\title{
Los Premios a la Investigación Científica de la UNAH: Un instrumento de estímulo y promoción de la actividad investigativa
}

Elsa Lily Caballero Zeitún*

Escribir sobre los Premios de Investigación Científica con los que la UNAH distingue a sus investigadores, hubiera sido una tarea menos comprometida si no gozara del honor de recibir éste reconocimiento. El entrenamiento en la investigación científica nos condiciona a una práctica disciplinada de discernimiento imparcial al momento de hacer una descripción y análisis de alguna situación; sin embargo, el hecho de ser partícipe vuelve más complicada la tarea ya que, a la descripción y significados que se pueden hacer del hecho en sí, se le suman y entrelazan los sentimientos por ser parte involucrada, en éste caso en particular, sentimientos especialmente agradables.

Recibir este premio es motivo de orgullo, satisfacción y muchas emociones más, que provocan esa profunda sensación de alegría que experimentamos en esos momentos sumamente agradables que la vida nos regala. Sin tener un orden específico, ya que los sentimientos no lo tienen, se agrupan y fluyen desbordadamente, esa profunda alegría se debe a que se nos reconoce públicamente méritos, capacidades y la contribución por descubrir, crear, inventar, innovar o renovar el conocimiento científico sobre la realidad desde alguna disciplina científica particular; sin duda alguna, con ello nos halagan el ego, que no está de más recordar, es una de las esencias humanas. Desde esta posición, en consecuencia, es muy poco lo que se puede decir sobre los premios de investigación de la UNAH, pues se resume, para los que no tenemos el arte de las letras mágicas, en una profunda alegría y lo único que podemos hacer es manifestar nuestro agradecimiento.

Un premio a la investigación científica tiene una larga trayectoria en la comunidad científica mundial y, en este caso, al tratarse de un premio que otorga la universidad a los investigadores que se ocupan de una actividad que es intrínseca a la academia, interesa destacar el proceso de institucionalización del premio y algunos de sus significados.

Universidad Nacional Autónoma de Honduras. Facultad de Ciencias Sociales. Maestría en Gestión Social Urbana.zeitunlily@yahoo.com 


\section{Institucionalización del premio de investigación científica de la UNAH.}

Del premio de investigación científica de la UNAH podemos decir que se trata de un proceso en el que destacan dos características, primero, que el reconocimiento institucional de los investigadores es reciente y segundo, que se ha ido perfeccionando como proceso de reconocimiento institucional. De acuerdo a la información proporcionada por el Dr. Javier Sánchez Midence, de la Dirección de Investigación Científica de la UNAH, éste premio tiene su antecedente en en el año 2006, cuando el Dr. Benjamín Henríquez, Director de Investigación Científica y la Dra. Dixiana de Vásquez, solicitaron a la Comisión de Transición instaurar el Premio a la trayectoria en investigación científica Dr. Salvador Moncada", en honor al ilustre científico mundial, candidato al Premio Nobel y que Honduras tuvo la suerte de ser su país natal, siendo los galardonados con éste reconocimiento, el Dr. Gustavo Pérez del área Físico Matemática y el Dr. Marco Tulio Medina, del áreas de Ciencias Biológicas y de la Salud.

A partir del año 2007, siendo la Directora de Investigación Científica, la socióloga Leticia Salomón, se crea e institucionaliza el Premio "Investigación Científica, UNAH", al establecer que será entregado anualmente en el marco del Congreso anual de Investigación Científica y que "el ganador/a será seleccionado por un Jurado de cinco miembros, integrado por la Vicerrectora Académica, la Directora de Investigación Científica y tres personalidades más nombradas por la Dirección de Investigación Científica" (Convocatoria Premio Investigación Científica UNAH, 2007). La Dirección de Investigación Científica hace el lanzamiento del Premio bajo el enunciado: "por el compromiso con el desarrollo científico y tecnológico, bajo el paradigma de pertinencia, relevancia, calidad y ética, convoca a los investigadores de la UNAH a participar en el concurso para el Premio Investigación Científica, UNAH 2007, a fin de reconocer la contribución que los investigadores de la UNAH brindan a la sociedad y el desarrollo científico, tecnológico y cultural" (Convocatoria Premio Investigación Científica UNAH, 2007).

En esta convocatoria, se estipula que el requisito es el ejercicio permanente y diverso de la investigación: "Se evaluarán las labores de Investigación que los y las investigadoras estén llevando a cabo o hayan efectuado durante su carrera universitaria en la UNAH. Para otorgar este premio no se considerará el trabajo docente, de acción social, labor administrativa ni de gestión. Sin embargo, se tomará en consideración toda la labor de investigación que se detalla en el trifolio de la Dirección: Criterios de clasificación de Investigadores. Todos los nominados deberán estar registrados como investigadores en la Dirección de Investigación Científica". 
El premio fue otorgado, en el 2007, al Dr. Cirilo Nelson, del área de ciencias biológicas; en el 2008, al Dr. Américo Reyes Ticas del área Psiquiatría; en el año 2009, a la Dra. Ivette Lorenzana del área de Microbiología; en el año 2010 el premio fue declarado desierto y en el 2011, obtiene el primer lugar en la categoría de profesor/investigador el Dr. Marco Tulio Medina; En 2011 se lanza un nuevo premio a las ideas para la innovación, en el cual resultan seleccionados Freddy Torres Mejía y Alejandro Colindres, en la categorías profesional y estudiantil, respectivamente (Dr. Javier Sánchez Midence, 2012, DICU).

Luego de cinco años de trayectoria del Premio Investigación Científica, UNAH, en el año 2012 se ampliaron las categorías de investigadores hasta llegar a definir cuatro; de igual forma en las diferentes convocatorias se ha ido ampliando la información sobre las bases con las cuales se otorga este premios. Para el año 2012 las categorías de premiación fueron cuatro. Ver cuadro No. 1.

Cuadro 1. Categorías del Premio de Investigación Científica, UNAH

\begin{tabular}{|c|c|}
\hline Categoría & Decripción \\
\hline Investigador con Larga Trayectoria & $\begin{array}{l}\text { Es el investigador que ha acumulado más de } 15 \text { años de } \\
\text { experiencia como investigador activo. Los años de } \\
\text { investigador deben ser acreditados como investigador } \\
\text { individual o grupal y en este caso puede ser como asistente, } \\
\text { investigador secundario y principal. Debe acreditar su } \\
\text { participación en investigaciones, su condición de coordinador } \\
\text { de proyectos de investigación y tener una gran cantidad de } \\
\text { publicaciones en forma de libros y/o de artículos en revistas } \\
\text { nacionales y extranjeras, consecuente con sus años de } \\
\text { investigador. }\end{array}$ \\
\hline Investigador en Consolidación & $\begin{array}{l}\text { Es el investigador con una experiencia acreditada de } 10 \text { a } 15 \\
\text { años como investigador activo. Los años de investigador } \\
\text { deben ser acreditados como investigador individual o grupal y } \\
\text { en este caso, como asistente o como investigador secundario } \\
\text { o principal y con una cantidad razonable de publicaciones a } \\
\text { nivel nacional e internacional, consecuente con sus años de } \\
\text { investigador. }\end{array}$ \\
\hline $\begin{array}{c}\text { Investigador en Formación, a Nivel } \\
\text { Profesional }\end{array}$ & $\begin{array}{l}\text { Es el investigador con suficiente experiencia ( } 5 \text { a10 años), } \\
\text { expresada en ejecución de proyectos de investigación y } \\
\text { publicaciones nacionales y/o internacionales a nivel individual } \\
\text { y/o grupal, consecuente con sus años de investigador. }\end{array}$ \\
\hline $\begin{array}{c}\text { Investigador en Formación, a Nivel } \\
\text { Estudiantil }\end{array}$ & $\begin{array}{l}\text { Es el estudiante de grado o postgrado con poca experiencia } \\
\text { como investigador (entre } 3 \text { y } 5 \text { años) pero que se ha } \\
\text { involucrado en proyectos de investigación como asistente en } \\
\text { alguna parte del proceso: recolección de datos, cálculo de } \\
\text { muestra, análisis, y ha participado en investigaciones y } \\
\text { publicaciones a nivel individual yo grupal. }\end{array}$ \\
\hline
\end{tabular}

Fuente: Convocatoria Premio Investigación Científica UNAH 2012, 2013. 
En la convocatoria se enuncia que "el premio constituye un reconocimiento a la trayectoria, actividades y méritos de los investigadores de la UNAH -profesionales y estudiantes, en las categorías correspondientes - por su contribución al desarrollo de la ciencia, tecnología e innovación, y al conocimiento y solución de los principales problemas del país, enalteciendo con ello a la universidad y al país" (Convocatoria Premio Investigación Científica UNAH, 2012).

Los criterios de evaluación son agrupados en dos categorías según su importancia para la selección de los/las ganadores/as los cuales aparecen en la publicación de la convocatoria. Ver Cuadro No. 2.

\section{Cuadro 2. Criterios de Evaluación para Entregar el Premio}

\begin{tabular}{|l|l}
\hline \multicolumn{1}{c}{ Criterio } & \multicolumn{1}{c}{ Decripción } \\
\hline Valoración Fundamental & $\begin{array}{l}\text { Autoría y Co-autoría de Libros nacionales e } \\
\text { internacionales con sello editorial. }\end{array}$ \\
\hline $\begin{array}{l}\text { Autoría o Co-autoría de artículos en Revistas } \\
\text { Científicas nacionales e internacionales. }\end{array}$ \\
\hline $\begin{array}{l}\text { Autoría o Co-autoría de investigaciones sin publicar, } \\
\text { con constancia de participación institucional en la que } \\
\text { se especifique su papel en el proceso. }\end{array}$ \\
\hline $\begin{array}{l}\text { Autoría y Co-autoría de Libros nacionales e } \\
\text { internacionales con sello editorial. }\end{array}$ \\
\hline Conferencias nacionales e internacionales. \\
\hline Ponencias nacionales e internacionales; \\
\hline Tesis de Maestría yoctorado; \\
\hline Asesorías de tesis de Maestría yoctorado; \\
\hline Pares revisores de revistas nacionales o extranjeras; \\
\hline Evaluadores de proyectos yavances de investigación; \\
\hline $\begin{array}{l}\text { Miembros de Consejo Asesor en investigación o de } \\
\text { Consejo Editorial de revistas científicas; }\end{array}$ \\
\hline $\begin{array}{l}\text { Cargos en instancias de investigación a nivel nacional, } \\
\text { regional e internacional. }\end{array}$ \\
\hline
\end{tabular}

Fuente: Convocatoria Premio Investigación Científica UNAH 2012, 2013.

Esta premiación cubre toda la escala de investigadores, desde los de mayor trayectoria hasta los que se están iniciando como investigadores.

Adicional a las cuatro categorías, se incluyen otros premios (Ciencia y Tecnología; Mejoramiento a la Calidad de Vida; Ideas para la Innovación Tecnológica, Categoría Profesional; Ideas para la Innovación Tecnológica, Categoría Estudiante y 
Reconocimiento Especial al Mérito en tesis doctoral a una becaria de investigación UNAH), con las que se distingue un aporte científico específico e igual importancia adquiere para la comunidad científica nacional el reconocimiento de méritos a tesis de postgrado que logran mención honorífica. Los nombres de los premiados se pueden apreciar en el Cuadro No. 3.

Cuadro 3. Premios Investigación Científica UNAH 2012

\begin{tabular}{|c|c|c|c|}
\hline No. & Categoría del Premio & $\begin{array}{c}\text { Nombre } \\
\text { del Premiado }\end{array}$ & Unidad Académica \\
\hline 1 & $\begin{array}{l}\text { Investigador de Larga } \\
\text { Trayectoria }\end{array}$ & Elsa Lily Caballero & $\begin{array}{l}\text { Facultad de Ciencias Sociales, } \\
\text { Maestría en Gestión Social } \\
\text { Urbana }\end{array}$ \\
\hline 2 & Investigador en Consolidación & Lelany Pineda & $\begin{array}{l}\text { Facultad de Ciencias, Escuela } \\
\text { de Microbiología }\end{array}$ \\
\hline 3 & $\begin{array}{c}\text { Investigador en } \\
\text { Formación/Profesional }\end{array}$ & Miguel Barahona & $\begin{array}{l}\text { Facultad de Humanidades y } \\
\text { Artes, Escuela de Letras }\end{array}$ \\
\hline 4 & $\begin{array}{c}\text { Investigador en } \\
\text { Formación/Estudiante }\end{array}$ & $\begin{array}{l}\text { Cynthia Rodríguez } \\
\text { Galo }\end{array}$ & $\begin{array}{l}\text { Facultad de Ciencias, Ma- } \\
\text { estría en Enfermedades } \\
\text { Zoonóticas e Infecciosas }\end{array}$ \\
\hline 5 & Ciencia y Tecnología & $\begin{array}{l}\text { - Gustavo Fontecha } \\
\text { - Wilfredo Sosa } \\
\text { - Irina Jovel } \\
\text { - Rosa Elena Mejía } \\
\text { - Engels Banegas }\end{array}$ & $\begin{array}{l}\text { Facultad de Ciencias, Escuela } \\
\text { de Microbiología }\end{array}$ \\
\hline 6 & $\begin{array}{c}\text { Mejoramiento a la Calidad de } \\
\text { Vida }\end{array}$ & $\begin{array}{l}\text { Ivette Lorenzana } \\
\text { Pineda }\end{array}$ & $\begin{array}{l}\text { Facultad de Ciencias, Escuela } \\
\text { de Microbiología }\end{array}$ \\
\hline 7 & $\begin{array}{c}\text { Ideas para la Innovación } \\
\text { Tecnológica, Categoría } \\
\text { Profesional }\end{array}$ & Yeny Carolina Carías & $\begin{array}{l}\text { Facultad de Ingeniería, Depar- } \\
\text { tamento de Ingeniería en Sis- } \\
\text { temas }\end{array}$ \\
\hline 8 & $\begin{array}{c}\text { Ideas para la Innovación } \\
\text { Tecnológica, Categoría } \\
\text { Estudiante }\end{array}$ & $\begin{array}{l}\text { Oscar Armando } \\
\text { Castillo }\end{array}$ & $\begin{array}{l}\text { Facultad de Ingeniería, Es- } \\
\text { cuela de Ingeniería Civil }\end{array}$ \\
\hline 9 & $\begin{array}{c}\text { Reconocimiento Especial al } \\
\text { Mérito en Tesis Doctoral a } \\
\text { una becaria de Investigación, } \\
\text { UNAH }\end{array}$ & Emilia Alduvín & $\begin{array}{l}\text { Facultad de Ciencias Médicas, } \\
\text { Posgrado en Salud Pública }\end{array}$ \\
\hline
\end{tabular}

Fuente: Publicación Premios Investigación Científica UNAH 2012

Un rasgo importante en el proceso de evolución del premio es la diferenciación de categorías de investigadores, que incluye a investigadores formados y dos categorías en formación (profesionales y estudiantes), con lo cual se abren 
posibilidades que incentivan a los investigadores universitarios para mejorar su práctica de investigación, siendo importante destacar de la convocatoria, el estímulo para que estudiantes universitarios incursionen en la investigación, con lo que la universidad da un mensaje positivo a la comunidad estudiantil para que inicien su proceso de formación como investigadores.

\section{Los Significados del Premio Investigación Científica UNAH}

En el contexto de un país que no necesariamente se ha caracterizado por incentivar el desarrollo de la ciencia y tecnología, la existencia del premio a los investigadores de la UNAH, posee varios significados. En primer lugar, que en la UNAH ha existido una comunidad de científicos, personas que ejercen el oficio de investigar para los que la investigación ha sido parte de la cotidianidad de su vida laboral y no una actividad esporádica o circunstancial, y que, desde un campo particular de la ciencia, construyen relaciones e interacciones dentro y fuera del país para contribuir con el pensamiento científico universal. De ello da cuenta el Catálogo de Investigadores que la Dirección de Investigación Científica empieza a publicar desde el año 2009. En segundo lugar, después de varias décadas en que a lo interno de la UNAH no se le daba ninguna importancia a la investigación como actividad académica, a partir del año 2006 se refuerza el proceso de institucionalización de la investigación para que llegue a ser parte central de la actividad universitaria.

La actividad científica se puede ejercer sin una estructura institucional especializada, aunque sea de forma personal y aislada. Sólo es necesario que existan personas comprometidas y entrenadas para este ejercicio profesional y que dediquen buena parte de su vida a la producción del conocimiento científico, pues la producción científica es un ejercicio sistemático que se logra con la acumulación de experiencia, la especialización temática y la rigurosidad metodológica.

Por su parte, los premios a la investigación científica sólo pueden otorgarse cuando existe un conjunto de normas y procedimientos que protejan la seriedad que debe tener, así como su continuidad, ya que estamos frente a la cúspide simbólica de la estructura y proceso institucional de la investigación y entre la comunidad científica mundial los premios a la investigación, son una institución en sí misma.

En la UNAH, que ha producido reconocidos/as y talentosos/as investigadores/as, es en los últimos años que se inicia el proceso de institucionalización de este premio con lo que la institución está dando muestras y mensajes claros de que la investigación científica dejará de ser solo una actividad de interés personal de 
quienes la ejercen, para convertirse en un interés institucional que vendrá a reforzar las capacidades acumuladas por esa comunidad de investigadores universitarios.

Otro aspecto importante no solo para los investigadores sino para el país en general, es que se dan a conocer los aportes a la investigación científica y que se conozca la cantidad y calidad de los investigadores con que cuenta la universidad. Con el premio a la investigación científica de la UNAH se logra hacer de público conocimiento lo que se investiga en la institución y los que se encargan de esta actividad, lo que le da especial relevancia ya que generalmente los trabajos de investigación quedan confinados en los círculos especializados de los investigadores y no necesariamente se difunden más allá que la publicación de un libro o la presentación de resultados en algún evento público de audiencia interesada; así, los trabajos de los investigadores se quedan entre los muros de su especialidad y con muchas dificultades salen de esos espacios, lo que no es tan bueno para la investigación y los investigadores, pues por un lado, se restringe el espacio de circulación del conocimiento y por otro, los investigadores no tenemos la retroalimentación necesaria para valorar la relevancia de nuestro trabajo y seguir perfeccionándolo.

Uno de los significados relevantes desde el punto de vista del incentivo a la producción científica, es premiar el esfuerzo de la investigación como ejercicio profesional. Al generar esta expectativa, se procura un espacio que alienta a los y las investigadoras a seguir trabajando, ahora con la posibilidad de obtener un reconocimiento público por los resultados del trabajo, quedando pendiente el reto de lograr la participación de profesores de todas las facultades de la UNAH.

Destaca en la última convocatoria al premio de investigación de la UNAH, la amplitud de la invitación. En un país que requiere del aporte científico de sus profesionales y donde no ha sido cultivado el incentivo a la producción científica, es un buen mensaje para los investigadores la existencia de una gama de premios a los cuales se puede acceder a través de un concurso, en vez de cerrar las oportunidades a una sola persona a ser premiada, se abren las opciones de que, además de las cuatro categorías de investigadores definidas, se hace reconocimiento de trabajos de investigación con otros criterios para reconocer méritos, pero que igualmente enriquecen la producción científica y tecnológica del país.

Finalmente, un elemento de mucha importancia es la inclusión en la convocatoria de premios de investigación a estudiantes universitarios; si premiar a investigadores formados 0 en formación es estratégico para la institución y el país, por la contribución que la UNAH hace desde los diferentes campos de la ciencia al 
esclarecimiento de los problemas y/o contribuir con soluciones a los mismos, incluir en el reconocimiento de méritos a los estudiantes, permite incluir dos significados más sobre la importancia y la trascendencia del premio.

La importancia del reconocimiento a jóvenes que desde su etapa formativa muestran inquietudes y destrezas para la investigación, es enorme y loable, pues esas incipientes muestras de interés ya no sólo son alentadas por un profesor investigador que descubre esos talentos, sino que la institución reconoce y fomenta el desarrollo de ese potencial, lo que hace que la trascendencia de este acto vaya mucho más allá del reconocimiento a estos jóvenes, sino que abre un enorme espacio - el semillero adecuado- para cultivar la creatividad, las preocupaciones intelectuales y el entrenamiento temprano en las pericias de la investigación de los futuros investigadores profesionales del país. A lo interno de la UNAH, un estímulo como éste podría llegar a tener un tremendo impacto en la calidad de la docencia, si llegara también a ser utilizado como criterio de escogencia de los futuros docentes universitarios, ya que, sin lugar a dudas, podemos afirmar que la docencia mejor calificada es la que se respalda en los hallazgos de la investigación.

La inclusión de otros premios específicos (ideas para la innovación, mejor investigación en los últimos dos años y mejor tesis apoyada por una beca de investigación), además de la incorporación de varias categorías al Premio UNAH a la Investigación, es un elemento fundamental para la motivación de las diferentes disciplinas científicas, como lo muestra la procedencia disciplinar de todos los galardonados de la UNAH en 2012. Con estos premios la UNAH se enaltece como institución, a la vez que enaltece a los investigadores, profesionales y estudiantes, y a las escuelas y facultades de las que proceden. 